\title{
Weak Convergence Theorem for Finding Fixed Points and Solution of Split Feasibility and Systems of Equilibrium Problems
}

\author{
Kamonrat Sombut and Somyot Plubtieng \\ Department of Mathematics, Faculty of Science, Naresuan University, Phitsanulok 65000, Thailand \\ Correspondence should be addressed to Somyot Plubtieng; somyotp@nu.ac.th
}

Received 15 October 2012; Accepted 13 December 2012

Academic Editor: Allan Peterson

Copyright (C) 2013 K. Sombut and S. Plubtieng. This is an open access article distributed under the Creative Commons Attribution License, which permits unrestricted use, distribution, and reproduction in any medium, provided the original work is properly cited.

\begin{abstract}
The purpose of this paper is to introduce an iterative algorithm for finding a common element of the set of fixed points of quasinonexpansive mappings and the solution of split feasibility problems (SFP) and systems of equilibrium problems (SEP) in Hilbert spaces. We prove that the sequences generated by the proposed algorithm converge weakly to a common element of the fixed points set of quasi-nonexpansive mappings and the solution of split feasibility problems and systems of equilibrium problems under mild conditions. Our main result improves and extends the recent ones announced by Ceng et al. (2012) and many others.
\end{abstract}

\section{Introduction}

Let $C$ be a nonempty closed convex subset of a real Hilbert space $H$. A mapping $T: C \rightarrow C$ is said to be nonexpansive if $\|T x-T y\| \leq\|x-y\|$ for all $x, y \in C$. Denote the set of fixed points of $T$ by $F(T)$. On the other hand, a mapping $T: C \rightarrow C$ is said to be quasi-nonexpansive if $F(T) \neq \emptyset$ and $\|T x-q\| \leq\|x-q\|$ for all $x \in C$ and $q \in F(T)$. If $T: C \rightarrow C$ is nonexpansive and the set $F(T)$ of fixed points of $T$ is nonempty, then $T$ is quasi-nonexpansive. Fixed point iterations process for nonexpansive mappings and quasinonexpansive mappings in Banach spaces including Mann and Ishikawa iterations process have been studied extensively by many authors to solve the nonlinear operator equations (see [1-4]).

Let $F$ be a bifunction of $C \times C$ into $\mathbb{R}$, where $\mathbb{R}$ is the set of real numbers. The equilibrium problem for $F: C \times C \rightarrow \mathbb{R}$ is to find $x \in C$ such that

$$
F(x, y) \geq 0 \quad \forall y \in C .
$$

The set of solutions of (1) is denoted by $\operatorname{EP}(F)$. Numerous problems in physics, optimization, and economics reduce to find a solution of (1) in Hilbert spaces; see, for instance,
Blum and Oettli [5], Flam and Antipin [6], and Moudafi [7]. Moreover, Flam and Antipin [6] introduced an iterative scheme of finding the best approximation to the solution of equilibrium problem, when $\mathrm{EP}(F)$ is nonempty, and proved a strong convergence theorem (see also in [8-11]). Let $F_{1}, F_{2}$ : $C \times C \rightarrow \mathbb{R}$ be two-monotone bifunction and $\lambda>0$ is a constant. Recently, Moudafi [12] considered the following of a system of equilibrium problem, denoting the set of solution of SEP by $\Omega$, for finding $\left(x^{*}, y^{*}\right) \in C \times C$ such that

$$
\begin{array}{ll}
\lambda F_{1}\left(x^{*}, z\right)+\left\langle y^{*}-x^{*}, x^{*}-z\right\rangle \geq 0, & \forall z \in C, \\
\lambda F_{2}\left(y^{*}, z\right)+\left\langle x^{*}-y^{*}, y^{*}-z\right\rangle \geq 0, & \forall z \in C .
\end{array}
$$

He also proved the weak convergence theorem of this problem (some related work can be found in $[13,14])$.

The split feasibility problem (SFP) in Hilbert spaces for modeling inverse problems which arise from phase retrievals and in medical image reconstruction was first introduced by Censor and Elfving [15] (see, e.g., [16, 17]). It has been found that the SFP can also be used to model the intensitymodulated radiation therapy (see $[18,19])$. In this work, the SFP is formulated as finding a point $x^{*}$ with the property

$$
x^{*} \in C, \quad A x^{*} \in Q \text {, }
$$


where $C$ and $Q$ are the nonempty closed convex subsets of the infinite-dimensional real Hilbert spaces $H_{1}$ and $H_{2}$, respectively, and $A \in B\left(H_{1}, H_{2}\right)$ (i.e., $A$ is a bounded linear operator from $H_{1}$ to $H_{2}$ ). Very recently, there are related works which we can find in $[16,18,20-26]$ and the references therein.

A special case of the SFP is called the convex constrained linear inverse problem (see [27]), that is, the problem to finding an element $x$ such that

$$
x \in C, \quad A x=b \in Q \text {. }
$$

In fact, it has been extensively investigated in the literature using the projected Landweber iterative method [27, 28]. Throughout this paper, we assume that the solution set $\Gamma$ of the SFP is nonempty.

Motivated and inspired by the regularization method and extragradient method due to Ceng et al. [29], we introduce and analyze an extragradient method with regularization for finding a common element of the fixed points set of quasinonexpansive mappings and the solution of split feasibility problems (SFP) and systems of equilibrium problems (SEP) in Hilbert spaces. Our results represent the improvement, extension, and development of the corresponding results in $[14,29]$.

\section{Preliminaries}

Let $H$ be a real Hilbert space with inner product $\langle\cdot, \cdot\rangle$ and norm $\|\cdot\|$, and let $C$ be a closed convex subset of $H$. We write $x_{n} \rightarrow x$ to indicate that the sequence $\left\{x_{n}\right\}$ converges weakly to $x$ and $x_{n} \rightarrow x$ to indicate that the sequence $\left\{x_{n}\right\}$ converges strongly to $x$. For every point $x \in H$, there exists a unique nearest point in $C$, denoted by $P_{C} x$, such that

$$
\left\|x-P_{C} x\right\|=\inf _{y \in C}\|x-y\| \leq\|x-y\| \quad \forall y \in C .
$$

$P_{C}$ is called the metric projection of $H$ onto $C$.

Some important properties of projections are gathered in the following proposition.

Proposition 1 (see [29]). For given $x \in H$ and $z \in C$ :

(i) $z=P_{C} x$ if and only if $\langle x-z, y-z\rangle \leq$ 0 , for all $y \in C$;

(ii) $z=P_{C} x$ if and only if $\|x-z\|^{2} \leq\|x-y\|^{2}-\| y-$ $z \|^{2}$, for all $y \in C$.

Definition 2 (see $[30,31])$. Let $T$ be a nonlinear operator with domain $D(T) \subseteq H$ and range $R(T) \subseteq H$, and let $\beta>0$ and $v>0$ be given constants. The operator $T$ is called

(a) monotone if

$$
\langle x-y, T x-T y\rangle \geq 0, \quad \forall x, y \in D(T)
$$

(b) $\beta$-strongly monotone if

$$
\langle x-y, T x-T y\rangle \geq \beta\|x-y\|^{2}, \quad \forall x, y \in D(T) ;
$$

(c) v-inverse strongly monotone $(v$-ism) if

$$
\langle x-y, T x-T y\rangle \geq v\|T x-T y\|^{2}, \quad \forall x, y \in D(T) .
$$

We can easily see that if $S$ is nonexpansive, then $I-S$ is monotone. It is also easy to see that a projection $P_{C}$ is a 1-ism.

Definition 3 (see [29]). A mapping $T: H \rightarrow H$ is said to be an averaged mapping if it can be written as the average of the identity $I$ and a nonexpansive mapping, that is,

$$
T \equiv(1-\alpha) I+\alpha S
$$

where $\alpha \in(0,1)$ and $S: H \rightarrow H$ is nonexpansive. More precisely, when (9) holds, we say that $T$ is $\alpha$-averaged. It is easly to see that if $T$ is an averaged mapping, then $T$ is nonexpansive.

Proposition 4 (see [20]). Let $T: H \rightarrow H$ be a given mapping. Then consider the following.

(i) $T$ is nonexpansive if and only if the complement $I-T$ is $(1 / 2)$-ism.

(ii) $T$ is averaged if and only if the complement $I-T$ is $v$-ism for some $v>1 / 2$. Indeed, for $\alpha \in(0,1), T$ is $\alpha$-averaged if and only if $I-T$ is $(1 / 2 \alpha)$-ism.

(iii) The composite of finitely many averaged mappings is averaged. That is, if each of the mappings $\left\{T_{i}\right\}_{N}^{i=1}$ is averaged, then so is the composite $T_{1} \circ T_{2} \circ \ldots \circ T_{N}$. In particular, if $T_{1}$ is $\alpha_{1}$-averaged and $T_{2}$ is $\alpha_{2}$-averaged, where $\alpha_{1}, \alpha_{2} \in(0,1)$, then the composite $T_{1} \circ T_{2}$ is $\alpha$ averaged, where $\alpha=\alpha_{1}+\alpha_{2}-\alpha_{1} \alpha_{2}$.

In this paper, we use an equilibrium bifunction $F: C \times$ $C \rightarrow \mathbb{R}$ for solving the equilibrium problems, let us assume that $F$ satisfies the following conditions:

(A1) $F(x, x)=0$ for all $x \in C$;

(A2) $F$ is monotone, that is, $F(x, y)+F(y, x) \leq 0$ for all $x, y \in C$;

(A3) for each $y \in C, x \mapsto F(x, y)$ is weakly upper semicontinuous;

(A4) for each $x \in C, y \mapsto F(x, y)$ is convex; semicontinuous.

Lemma 5 (see [6]). Assume that $F: C \times C \rightarrow \mathbf{R}$ satisfies (A1)(A4). For $r>0$ and $x \in H$, define a mapping $T_{r}: H \rightarrow C$ as follows:

$$
T_{r}(x)=\left\{z \in C: F(z, y)+\frac{1}{r}\langle y-z, z-x\rangle \geq 0, \forall y \in C\right\},
$$

for all $z \in H$. Then, the following hold:

(i) $T_{r}$ is single-valued;

(ii) $T_{r}$ is firmly nonexpansive, that is, for any $x, y \in$ $H,\left\|T_{r} x-T_{r} y\right\|^{2} \leq\left\langle T_{r} x-T_{r} y, x-y\right\rangle$; 
(iii) $F\left(T_{r}\right)=E P(F)$;

(iv) $E P(F)$ is closed and convex.

Lemma 6 (see [32]). Let $C$ be a closed convex subset of a real Hilbert space $H$. Let $F_{1}$ and $F_{2}$ be two mappings from $C \times C \rightarrow \mathbf{R}$ satisfying (A1)-(A4) and let $T_{1, \lambda}$ and $T_{2, \mu}$ are defined as in Lemma 5 associated to $F_{1}$ and $F_{2}$, respectively. For given $x^{*}, y^{*} \in C,\left(x^{*}, y^{*}\right)$ is a solution of problem (2) if and only if $x^{*}$ is a fixed point of the mapping $G: C \rightarrow C$ defined by

$$
G(x)=T_{1, \lambda}\left(T_{2, \mu} x\right), \quad \forall x \in C,
$$

where $y^{*}=T_{2, \mu} x^{*}$.

Lemma 7 (see [33]). Let $\left\{a_{n}\right\}_{n=1}^{\infty},\left\{b_{n}\right\}_{n=1}^{\infty}$ and $\left\{\delta_{n}\right\}_{n=1}^{\infty}$ be sequences of nonnegative real numbers satisfying the inequality

$$
a_{n+1} \leq\left(1+\delta_{n}\right) a_{n}+b_{n}, \quad \forall n \geq 1 .
$$

If $\sum_{n=1}^{\infty} \delta_{n}<\infty$ and $\sum_{n=1}^{\infty} b_{n}<\infty$, then $\lim _{n \rightarrow \infty} a_{n}$ exists. If, in addition, $\left\{a_{n}\right\}_{n=1}^{\infty}$ has a subsequence which converges to zero, then $\lim _{n \rightarrow \infty} a_{n}=0$.

\section{Weak Convergence Theorem}

In this section, we prove a weak convergence theorem by an extragradient methods for finding a common element of the fixed points set of quasi-nonexpansive mappings and the solution of split feasibility problems and systems of equilibrium problems in Hilbert spaces. The function $f$ : $H \rightarrow \mathbb{R}$ is a continuous differentiable function with the minimization problem given by

$$
\min _{x \in C} f(x):=\frac{1}{2}\left\|A x-P_{Q} A x\right\|^{2} .
$$

In 2010, Xu [17] considered the following Tikhonov regularized problem:

$$
\min _{x \in C} f_{\alpha}(x):=\frac{1}{2}\left\|A x-P_{\mathrm{Q}} A x\right\|^{2}+\frac{1}{2} \alpha\|x\|^{2}
$$

where $\alpha>0$ is the regularization parameter. The gradient given by

$$
\nabla f_{\alpha}(x)=\nabla f(x)+\alpha I=A^{*}\left(I-P_{\mathrm{Q}}\right) A+\alpha I
$$

is $\left(\alpha+\|A\|^{2}\right)$-Lipschitz continuous and $\alpha$-strongly monotone (see [29] for the details).

Lemma 8 (see [17, 29]). The following hold:

(i) $\Gamma=F\left(P_{C}(I-\lambda \nabla f)\right)=V I(C, \nabla f)$ for any $\lambda>0$, where $F\left(P_{C}(I-\lambda \nabla f)\right)$ and $V I(C, \nabla f)$ denote the set of fixed points of $P_{C}(I-\lambda \nabla f)$ and the solution set of VIP;

(ii) $P_{C}\left(I-\lambda \nabla f_{\alpha}\right)$ is $\xi$-averaged for each $\lambda \in(0,2 /(\alpha+$ $\left.\left.\|A\|^{2}\right)\right)$, where $\xi=\left(2+\lambda\left(\alpha+\|A\|^{2}\right)\right) / 4$.
Theorem 9. Let $C$ be a nonempty closed convex subset in a real Hilbert space $H$. Let $\mu>0, F_{1}$ and $F_{2}$ be two bifunctions from $C \times C \rightarrow \mathbb{R}$ satisfying $(A 1)-(A 4)$. Let $S$ be a quasinonexpansive mapping of $C$ into itself such that $I-S$ be demiclosed at zero, that is, if $\left\{w_{n}\right\} \subset C, w_{n} \rightarrow w$ and $(I-$ $S) w_{n} \rightarrow 0$, then $w \in F(S)$, with $F(S) \cap \Gamma \cap \Omega \neq \emptyset$. Let $\left\{x_{n}\right\},\left\{y_{n}\right\}$, $\left\{z_{n}\right\}$, and $\left\{w_{n}\right\}$ be the sequence in $C$ generated by the following extragradient algorithm:

$$
\begin{gathered}
x_{0}=x \in C, \\
w_{n} \in C ; \quad F_{2}\left(w_{n}, z\right)+\varphi(z)-\varphi\left(w_{n}\right) \\
+\frac{1}{\mu}\left\langle z-w_{n}, w_{n}-x_{n}\right\rangle \geq 0, \quad \forall z \in C, \\
z_{n} \in C ; \quad F_{1}\left(z_{n}, z\right)+\varphi(z)-\varphi\left(z_{n}\right) \\
+\frac{1}{\mu}\left\langle z-z_{n}, z_{n}-w_{n}\right\rangle \geq 0, \quad \forall z \in C, \\
y_{n}=P_{C}\left(I-\lambda_{n} \nabla f_{\alpha_{n}}\right) z_{n}, \\
x_{n+1}=\beta_{n} x_{n}+\left(1-\beta_{n}\right) S P_{C}\left(x_{n}-\lambda_{n} \nabla f_{\alpha_{n}}\left(y_{n}\right)\right),
\end{gathered}
$$

where $\sum_{n=0}^{\infty} \alpha_{n}<\infty,\left\{\lambda_{n}\right\} \subset[a, b]$ for some $a, b \in\left(0,1 /\|A\|^{2}\right)$ and $\left\{\beta_{n}\right\} \subset[c, d]$ for some $c, d \in(0,1)$. Then, the sequences $\left\{x_{n}\right\}$ and $\left\{y_{n}\right\}$ converge weakly to an element $\tilde{x} \in F(S) \cap \Gamma \cap \Omega$.

Proof. By Lemma 8, we have that $P_{C}\left(I-\lambda \nabla f_{\alpha}\right)$ is $\xi$-averaged for each $\lambda \in\left(0,2 /\left(\alpha+\|A\|^{2}\right)\right)$, where $\xi=\left(2+\lambda\left(\alpha+\|A\|^{2}\right)\right) / 4$. Hence, by Proposition 2.4, $P_{C}\left(I-\lambda \nabla f_{\alpha}\right)$ is nonexpansive. From $\left\{\lambda_{n}\right\} \subset[a, b]$ and $a, b \in\left(0,1 /\|A\|^{2}\right)$, we have $a \leq$ $\inf _{n \geq 0} \lambda_{n} \leq \sup _{n \geq 0} \lambda_{n} \leq b<1 /\|A\|^{2}=\lim _{n \rightarrow \infty} 1 /\left(\alpha_{n}+\|A\|^{2}\right)$. Without loss of generality, we assume that $a \leq \inf _{n \geq 0} \lambda_{n} \leq$ $\sup _{n \geq 0} \lambda_{n} \leq b<1 /\left(\alpha_{n}+\|A\|^{2}\right)$, for all $n \geq 0$. Hence, for each $n \geq 0, P_{C}\left(I-\lambda_{n} \nabla f_{\alpha_{n}}\right)$ is $\xi_{n}$-averaged with

$$
\begin{aligned}
\xi_{n} & =\frac{1}{2}+\frac{\lambda_{n}\left(\alpha_{n}+\|A\|^{2}\right)}{2}-\frac{1}{2} \cdot \frac{\lambda_{n}\left(\alpha_{n}+\|A\|^{2}\right)}{2} \\
& =\frac{2+\lambda_{n}\left(\alpha_{n}+\|A\|^{2}\right)}{4} \in(0,1) .
\end{aligned}
$$

This implies that $P_{C}\left(I-\lambda_{n} \nabla f_{\alpha_{n}}\right)$ is nonexpansive for all $n \geq 0$.

Next, we show that the sequence $\left\{x_{n}\right\}$ is bounded. Indeed, take a fixed $p \in F(S) \cap \Gamma \cap \Omega$ arbitrarily. Let $T_{1, \mu}$ and $T_{2, \mu}$ be defined as in Lemma 5 associated to $F_{1}$ and $F_{2}$, respectively. Thus, we get $p=S p=S P_{C}(p)$, for all $n \geq$ $0, p=P_{C}(I-\lambda \nabla f) p$, for all $\lambda \in\left(0,2 /\|A\|^{2}\right)$ and $p=$ $T_{1, \mu}\left(T_{2, \mu} p\right)$, for all $\mu>0$. Put $y^{*}=T_{2, \mu} p, z_{n}=T_{1, \mu} w_{n}$ and $w_{n}=T_{2, \mu} x_{n}$. From (29), we have

$$
\begin{aligned}
\left\|y_{n}-p\right\| & =\left\|P_{C}\left(I-\lambda_{n} \nabla f_{\alpha_{n}}\right) z_{n}-P_{C}\left(I-\lambda_{n} \nabla f\right) p\right\| \\
& \leq\left\|P_{C}\left(I-\lambda_{n} \nabla f_{\alpha_{n}}\right) z_{n}-P_{C}\left(I-\lambda_{n} \nabla f_{\alpha_{n}}\right) p\right\|
\end{aligned}
$$




$$
\begin{aligned}
& +\left\|P_{C}\left(I-\lambda_{n} \nabla f_{\alpha_{n}}\right) p-P_{C}\left(I-\lambda_{n} \nabla f\right) p\right\| \\
\leq & \left\|z_{n}-p\right\|+\left\|\left(I-\lambda_{n} \nabla f_{\alpha_{n}}\right) p-\left(I-\lambda_{n} \nabla f\right) p\right\| \\
= & \left\|z_{n}-p\right\|+\left\|\lambda_{n} p\left(\nabla f-\nabla f_{\alpha_{n}}\right)\right\| \\
= & \left\|z_{n}-p\right\|+\left\|\lambda_{n} \alpha_{n} p\right\| \\
= & \left\|z_{n}-p\right\|+\lambda_{n} \alpha_{n}\|p\| .
\end{aligned}
$$

This implies that $\left\|z_{n}-p\right\|=\left\|T_{1, \mu} w_{n}-T_{1, \mu} y^{*}\right\| \leq\left\|w_{n}-y^{*}\right\|=$ $\left\|T_{2, \mu} x_{n}-T_{2, \mu} p\right\| \leq\left\|x_{n}-p\right\|$. Thus, we obtain $\left\|y_{n}-p\right\| \leq$ $\left\|x_{n}-p\right\|+\lambda_{n} \alpha_{n}\|p\|$. Put $l_{n}=P_{C}\left(x_{n}-\lambda_{n} \nabla f_{\alpha_{n}}\left(y_{n}\right)\right)$ for each $n \geq 0$. Then, by Proposition 1(ii), we have

$$
\begin{aligned}
\left\|l_{n}-p\right\|^{2} \leq & \left\|x_{n}-\lambda_{n} \nabla f_{\alpha_{n}}\left(y_{n}\right)-p\right\|^{2} \\
& -\left\|x_{n}-\lambda_{n} \nabla f_{\alpha_{n}}\left(y_{n}\right)-l_{n}\right\|^{2} \\
= & \left\|x_{n}-p\right\|^{2}-\left\|x_{n}-l_{n}\right\|^{2}+2 \lambda_{n}\left\langle\nabla f_{\alpha_{n}}\left(y_{n}\right), p-l_{n}\right\rangle \\
\leq & \left\|x_{n}-p\right\|^{2}-\left\|x_{n}-l_{n}\right\|^{2} \\
& +2 \lambda_{n}\left[\left\langle\nabla f_{\alpha_{n}}\left(y_{n}\right), y_{n}-l_{n}\right\rangle+\left\langle\nabla f_{\alpha_{n}} p, p-y_{n}\right\rangle\right] \\
\leq & \left\|x_{n}-p\right\|^{2}-\left\|x_{n}-l_{n}\right\|^{2} \\
& +2 \lambda_{n}\left[\alpha_{n}\left\langle p, p-y_{n}\right\rangle+\left\langle\nabla f_{\alpha_{n}}\left(y_{n}\right), y_{n}-l_{n}\right\rangle\right] \\
\leq & \left\|x_{n}-p\right\|^{2}-\left\|x_{n}-y_{n}\right\|^{2} \\
& -2\left\langle x_{n}-y_{n}, y_{n}-l_{n}\right\rangle-\left\|y_{n}-l_{n}\right\|^{2} \\
& +2 \lambda_{n}\left[\alpha_{n}\left\langle p, p-y_{n}\right\rangle+\left\langle\nabla f_{\alpha_{n}}\left(y_{n}\right), y_{n}-l_{n}\right\rangle\right] \\
= & \left\|x_{n}-p\right\|^{2}-\left\|x_{n}-y_{n}\right\|^{2}-\left\|y_{n}-l_{n}\right\|^{2} \\
& +2\left\langle x_{n}-\lambda_{n} \nabla f_{\alpha_{n}}\left(y_{n}\right)-y_{n}, l_{n}-y_{n}\right\rangle \\
& +2 \lambda_{n} \alpha_{n}\left\langle p, p-y_{n}\right\rangle .
\end{aligned}
$$

Hence, by Proposition 1(i), we have

$$
\begin{aligned}
\left\langle x_{n}\right. & \left.-\lambda_{n} \nabla f_{\alpha_{n}}\left(y_{n}\right)-y_{n}, l_{n}-y_{n}\right\rangle \\
= & \left\langle x_{n}-\lambda_{n} \nabla f_{\alpha_{n}}\left(x_{n}\right)-y_{n}, l_{n}-y_{n}\right\rangle \\
& +\left\langle\lambda_{n} \nabla f_{\alpha_{n}}\left(x_{n}\right)-\lambda_{n} \nabla f_{\alpha_{n}}\left(y_{n}\right), l_{n}-y_{n}\right\rangle \\
\leq & \left\langle\lambda_{n} \nabla f_{\alpha_{n}}\left(x_{n}\right)-\lambda_{n} \nabla f_{\alpha_{n}}\left(y_{n}\right), l_{n}-y_{n}\right\rangle \\
\leq & \lambda_{n}\left\|\nabla f_{\alpha_{n}}\left(x_{n}\right)-\lambda_{n} \nabla f_{\alpha_{n}}\left(y_{n}\right)\right\|\left\|l_{n}-y_{n}\right\| \\
\leq & \lambda_{n}\left(\alpha_{n}+\|A\|^{2}\right)\left\|x_{n}-y_{n}\right\|\left\|l_{n}-y_{n}\right\| .
\end{aligned}
$$

So, we have

$$
\begin{aligned}
\left\|l_{n}-p\right\|^{2} \leq & \left\|x_{n}-p\right\|^{2}-\left\|x_{n}-y_{n}\right\|^{2}-\left\|y_{n}-l_{n}\right\|^{2} \\
& +2 \lambda_{n}\left(\alpha_{n}+\|A\|^{2}\right)\left\|x_{n}-y_{n}\right\|\left\|l_{n}-y_{n}\right\| \\
& +2 \lambda_{n} \alpha_{n}\|p\|\left\|p-y_{n}\right\| \\
\leq & \left\|x_{n}-p\right\|^{2}-\left\|x_{n}-y_{n}\right\|^{2}-\left\|y_{n}-l_{n}\right\|^{2} \\
& +\lambda_{n}^{2}\left(\alpha_{n}+\|A\|^{2}\right)^{2}\left\|x_{n}-y_{n}\right\|^{2}+\left\|l_{n}-y_{n}\right\|^{2} \\
& +2 \lambda_{n} \alpha_{n}\|p\|\left\|p-y_{n}\right\| \\
= & \left\|x_{n}-p\right\|^{2}+2 \lambda_{n} \alpha_{n}\|p\|\left\|p-y_{n}\right\| \\
& +\left[\lambda_{n}^{2}\left(\alpha_{n}+\|A\|^{2}\right)^{2}-1\right]\left\|x_{n}-y_{n}\right\|^{2} \\
\leq & \left\|x_{n}-p\right\|^{2}+2 \lambda_{n} \alpha_{n}\|p\|\left\|p-y_{n}\right\| \\
\leq & \left\|x_{n}-p\right\|^{2}+2 \lambda_{n} \alpha_{n}\|p\|\left[\left\|z_{n}-p\right\|+\lambda_{n} \alpha_{n}\|p\|\right] \\
\leq & \left\|x_{n}-p\right\|^{2}+2 \lambda_{n} \alpha_{n}\|p\|\left[\left\|x_{n}-p\right\|+\lambda_{n} \alpha_{n}\|p\|\right] .
\end{aligned}
$$

Then, from the last inequality we conclude that

$$
\begin{aligned}
\left\|x_{n+1}-p\right\|^{2} & \\
= & \left\|\beta_{n} x_{n}+\left(1-\beta_{n}\right) S\left(l_{n}\right)-p\right\|^{2} \\
\leq & \beta_{n}\left\|x_{n}-p\right\|^{2}+\left(1-\beta_{n}\right)\left\|S\left(l_{n}\right)-p\right\|^{2} \\
& -\beta_{n}\left(1-\beta_{n}\right)\left\|x_{n}-S\left(l_{n}\right)\right\|^{2} \\
\leq & \beta_{n}\left\|x_{n}-p\right\|^{2}+\left(1-\beta_{n}\right)\left\|l_{n}-p\right\|^{2} \\
& -\beta_{n}\left(1-\beta_{n}\right)\left\|x_{n}-S\left(l_{n}\right)\right\|^{2} \\
\leq & \beta_{n}\left\|x_{n}-p\right\|^{2} \\
& +\left(1-\beta_{n}\right)\left[\left\|x_{n}-p\right\|^{2}+2 \lambda_{n} \alpha_{n}\|p\|\left\|p-y_{n}\right\|\right. \\
& \left.\quad+\left[\lambda_{n}^{2}\left(\alpha_{n}+\|A\|^{2}\right)^{2}-1\right]\left\|x_{n}-y_{n}\right\|^{2}\right] \\
& -\beta_{n}\left(1-\beta_{n}\right)\left\|x_{n}-S\left(l_{n}\right)\right\|^{2} \\
\leq & \left\|x_{n}-p\right\|^{2}+2 \lambda_{n} \alpha_{n}\|p\|\left\|p-y_{n}\right\| \\
& +\left(1-\beta_{n}\right)\left(\lambda_{n}^{2}\left(\alpha_{n}+\|A\|^{2}\right)^{2}-1\right)\left\|x_{n}-y_{n}\right\|^{2} \\
& -\beta_{n}\left(1-\beta_{n}\right)\left\|x_{n}-S\left(l_{n}\right)\right\|^{2} \\
\leq & \left\|x_{n}-p\right\|^{2}+\alpha_{n}\left(\lambda_{n}^{2}\|p\|^{2}+\left\|p-y_{n}\right\|^{2}\right) \\
& +\left(1-\beta_{n}\right)\left(\lambda_{n}^{2}\left(\alpha_{n}+\|A\|^{2}\right)^{2}-1\right)\left\|x_{n}-y_{n}\right\|^{2} \\
& -\beta_{n}\left(1-\beta_{n}\right)\left\|x_{n}-S\left(l_{n}\right)\right\|^{2}
\end{aligned}
$$




$$
\begin{aligned}
\leq & \left\|x_{n}-p\right\|^{2} \\
& +\alpha_{n}\left[\lambda_{n}^{2}\|p\|^{2}+\left(\left\|x_{n}-p\right\|+\lambda_{n} \alpha_{n}\|p\|\right)^{2}\right] \\
& +\left(1-\beta_{n}\right)\left(\lambda_{n}^{2}\left(\alpha_{n}+\|A\|^{2}\right)^{2}-1\right)\left\|x_{n}-y_{n}\right\|^{2} \\
& -\beta_{n}\left(1-\beta_{n}\right)\left\|x_{n}-S\left(l_{n}\right)\right\|^{2} \\
\leq & \left\|x_{n}-p\right\|^{2} \\
& +\alpha_{n}\left[\lambda_{n}^{2}\|p\|^{2}+2\left\|x_{n}-p\right\|^{2}+2 \lambda_{n}^{2} \alpha_{n}^{2}\|p\|^{2}\right] \\
& +\left(1-\beta_{n}\right)\left(\lambda_{n}^{2}\left(\alpha_{n}+\|A\|^{2}\right)^{2}-1\right)\left\|x_{n}-y_{n}\right\|^{2} \\
& -\beta_{n}\left(1-\beta_{n}\right)\left\|x_{n}-S\left(l_{n}\right)\right\|^{2} \\
= & \left(1+2 \alpha_{n}\right)\left\|x_{n}-p\right\|^{2}+\alpha_{n} \lambda_{n}^{2}\|p\|^{2}\left(1+2 \alpha_{n}^{2}\right) \\
& +\left(1-\beta_{n}\right)\left(\lambda_{n}^{2}\left(\alpha_{n}+\|A\|^{2}\right)^{2}-1\right)\left\|x_{n}-y_{n}\right\|^{2} \\
& -\beta_{n}\left(1-\beta_{n}\right)\left\|x_{n}-S\left(l_{n}\right)\right\|^{2} \\
\leq & \left(1+2 \alpha_{n}\right)\left\|x_{n}-p\right\|^{2}+\alpha_{n} \lambda_{n}^{2}\|p\|^{2}\left(1+2 \alpha_{n}^{2}\right) \\
= & \left(1+\delta_{n}\right)\left\|x_{n}-p\right\|^{2}+b_{n},
\end{aligned}
$$

where $\delta_{n}=2 \alpha_{n}$ and $b_{n}=\alpha_{n} \lambda_{n}^{2}\|p\|^{2}\left(1+2 \alpha_{n}^{2}\right)$. Since $\sum_{n=0}^{\infty} \alpha_{n}<$ $\infty$ and $\left\{\lambda_{n}\right\} \subset[a, b]$ for some $a, b \in\left(0,1 /\|A\|^{2}\right)$, we conclude that $\sum_{n=0}^{\infty} \delta_{n}<\infty$ and $\sum_{n=0}^{\infty} b_{n}<\infty$. Therefore, by Lemma 7 , we note that $\lim _{n \rightarrow \infty}\left\|x_{n}-p\right\|$ exists for each $p \in F(S) \cap \Gamma \cap$ $\Omega$ and hence the sequences $\left\{x_{n}\right\},\left\{l_{n}\right\},\left\{y_{n}\right\},\left\{z_{n}\right\}$, and $\left\{w_{n}\right\}$ are bounded. From (22), we also obtain

$$
\begin{aligned}
(1-d) & \left(1-b^{2}\left(\alpha_{n}+\|A\|^{2}\right)^{2}\right)\left\|x_{n}-y_{n}\right\|^{2} \\
& +c(1-d)\left\|x_{n}-S\left(l_{n}\right)\right\|^{2} \\
\leq & \left(1-\beta_{n}\right)\left(1-\lambda_{n}^{2}\left(\alpha_{n}+\|A\|^{2}\right)^{2}\right)\left\|x_{n}-y_{n}\right\|^{2} \\
& +\beta_{n}\left(1-\beta_{n}\right)\left\|x_{n}-S\left(l_{n}\right)\right\|^{2} \\
\leq & \left(1+2 \alpha_{n}\right)\left\|x_{n}-p\right\|^{2}-\left\|x_{n+1}-p\right\|^{2} \\
& +\alpha_{n} \lambda_{n}^{2}\|p\|^{2}\left(1+2 \alpha_{n}^{2}\right)
\end{aligned}
$$

where $\left\{\lambda_{n}\right\} \subset[a, b]$ and $\left\{\beta_{n}\right\} \subset[c, d]$. Since $\lim _{n \rightarrow \infty}\left\|x_{n}-p\right\|$ exists and $\alpha_{n} \rightarrow 0$, it follows that

$$
\begin{aligned}
\lim _{n \rightarrow \infty}\left\|x_{n}-y_{n}\right\| & =\lim _{n \rightarrow \infty}\left\|x_{n}-S\left(l_{n}\right)\right\| \\
& \leq \lim _{n \rightarrow \infty}\left[\left\|x_{n}-p\right\|+\left\|p-S\left(l_{n}\right)\right\|\right] \\
& \leq \lim _{n \rightarrow \infty}\left[\left\|x_{n}-p\right\|+\left\|p-l_{n}\right\|\right]=0 .
\end{aligned}
$$

Similarly, from inequality (22), we have

$$
\begin{aligned}
(1-d) & \left(1-b^{2}\left(\alpha_{n}+\|A\|^{2}\right)^{2}\right)\left\|x_{n}-z_{n}\right\|^{2} \\
& +c(1-d)\left\|x_{n}-S\left(l_{n}\right)\right\|^{2} \\
\leq & \left(1-\beta_{n}\right)\left(1-\lambda_{n}^{2}\left(\alpha_{n}+\|A\|^{2}\right)^{2}\right)\left\|x_{n}-z_{n}\right\|^{2} \\
& +\beta_{n}\left(1-\beta_{n}\right)\left\|x_{n}-S\left(l_{n}\right)\right\|^{2} \\
\leq & \left(1+\alpha_{n}\right)\left\|x_{n}-p\right\|^{2}-\left\|x_{n+1}-p\right\|^{2} \\
& +\alpha_{n} \lambda_{n}^{2}\|p\|^{2},
\end{aligned}
$$

where $\left\{\lambda_{n}\right\} \subset[a, b]$ and $\left\{\beta_{n}\right\} \subset[c, d]$. Since $\lim _{n \rightarrow \infty}\left\|x_{n}-p\right\|$ exists and $\alpha_{n} \rightarrow 0$, we obtain

$$
\lim _{n \rightarrow \infty}\left\|x_{n}-z_{n}\right\|=\lim _{n \rightarrow \infty}\left\|x_{n}-S\left(l_{n}\right)\right\|=0 .
$$

Moreover, we note that

$$
\begin{aligned}
\left\|y_{n}-l_{n}\right\| & =\left\|P_{C}\left(z_{n}-\lambda_{n} \nabla f_{\alpha_{n}} z_{n}\right)-P_{C}\left(x_{n}-\lambda_{n} \nabla f_{\alpha_{n}}\left(y_{n}\right)\right)\right\| \\
& \leq\left\|\left(z_{n}-\lambda_{n} \nabla f_{\alpha_{n}}\left(z_{n}\right)\right)-\left(x_{n}-\lambda_{n} \nabla f_{\alpha_{n}}\left(y_{n}\right)\right)\right\| \\
& =\left\|z_{n}-x_{n}-\left(\lambda_{n} \nabla f_{\alpha_{n}}\left(z_{n}\right)-\lambda_{n} \nabla f_{\alpha_{n}}\left(y_{n}\right)\right)\right\| \\
& \leq\left\|z_{n}-x_{n}\right\|+\left\|\lambda_{n} \nabla f_{\alpha_{n}}\left(z_{n}\right)-\lambda_{n} \nabla f_{\alpha_{n}}\left(y_{n}\right)\right\| \\
& \leq\left\|z_{n}-x_{n}\right\|+\lambda_{n}\left[\left\|\nabla f_{\alpha_{n}}\left(z_{n}\right)-\nabla f_{\alpha_{n}}\left(x_{n}\right)\right\|\right. \\
& \left.+\left\|\nabla f_{\alpha_{n}}\left(x_{n}\right)-\nabla f_{\alpha_{n}}\left(y_{n}\right)\right\|\right] \\
\leq & +\left\|z_{n}-x_{n}\right\|+\lambda_{n}\left[\left(\alpha_{n}+\|A\|^{2}\right)\left\|x_{n}-y_{n}\right\|\right. \\
& \left.\left.+z_{\alpha_{n}}\right)-\nabla f_{\alpha_{n}}\left(x_{n}\right) \|\right] .
\end{aligned}
$$

From (26) and $\alpha_{n} \rightarrow 0$, it is implied that

$$
\lim _{n \rightarrow \infty}\left\|y_{n}-l_{n}\right\|=0
$$

Note that $\left\|l_{n}-S\left(l_{n}\right)\right\| \leq\left\|l_{n}-y_{n}\right\|+\left\|y_{n}-x_{n}\right\|+\left\|x_{n}-S\left(l_{n}\right)\right\|$. This together with (24) and (28) implies that $\lim _{n \rightarrow \infty} \| l_{n}-$ $S\left(l_{n}\right) \|=0$. Also, from $\left\|x_{n}-l_{n}\right\| \leq\left\|x_{n}-y_{n}\right\|+\left\|y_{n}-l_{n}\right\|$, it follows that $\lim _{n \rightarrow \infty}\left\|x_{n}-l_{n}\right\|=0$. Since $\nabla f=A^{*}(I-$ $\left.P_{C}\right) A$ is a Lipschitz condition, where $A^{*}$ is the adjoint of $A$, we have $\lim _{n \rightarrow \infty}\left\|\nabla f\left(y_{n}\right)-\nabla f\left(l_{n}\right)\right\|=0$. Since $\left\{x_{n}\right\}$ is a bounded sequence, there exists a subsequence $\left\{x_{n_{j}}\right\}$ of $\left\{x_{n}\right\}$ that converges weakly to some $\widehat{x}$.

Next, we show that $\hat{x} \in \Gamma$. Since $\left\|x_{n}-l_{n}\right\| \rightarrow 0$ and $\| y_{n}-$ $l_{n} \| \rightarrow 0$, it is known that $l_{n_{i}} \rightarrow \widehat{x}$ and $y_{n_{i}} \rightarrow \widehat{x}$. Let $T: H \rightarrow$ $2^{H}$ be a set value mappings defined by

$$
T v= \begin{cases}\nabla f(v)+N_{C} v & \text { if } v \in C, \\ \emptyset & \text { if } v \notin C,\end{cases}
$$

where $N_{C} v=\left\{w \in H_{1}:\langle v-u, w\rangle \geq 0\right.$, for all $\left.u \in C\right\}$. Hence, by [34], $T$ is maximal monotone and $0 \in T v$ if and 
only if $v \in \operatorname{VI}(C, \nabla f)$. Let $(v, w) \in G(T)$. Then we have $w \in$ $T v=\nabla f(v)+N_{C} v$ and hence $w-\nabla f(v) \in N_{C} v$. So, we obtain $\langle v-u, w-\nabla f(v)\rangle \geq 0$, for all $u \in C$. On the other hand, from $l_{n}=P_{C}\left(x_{n}-\lambda_{n} \nabla f_{\alpha_{n}}\left(y_{n}\right)\right)$ and $v \in C$, we have

$$
\left\langle x_{n}-\lambda_{n} \nabla f_{\alpha_{n}}\left(y_{n}\right)-l_{n}, l_{n}-v\right\rangle \geq 0
$$

and hence

$$
\left\langle v-l_{n}, \frac{l_{n}-x_{n}}{\lambda_{n}}+\nabla f_{\alpha_{n}}\left(y_{n}\right)\right\rangle \geq 0 .
$$

Therefore, from $w-\nabla f(v) \in N_{C} v$ and $l_{n_{i}} \in C$, we get

$$
\begin{aligned}
\langle v- & \left.l_{n_{i}}, w\right\rangle \\
\geq & \left\langle v-l_{n_{i}}, \nabla f(v)\right\rangle \\
\geq & \left\langle v-l_{n_{i}}, \nabla f(v)\right\rangle \\
& -\left\langle v-l_{n_{i}}, \frac{l_{n_{i}}-x_{n_{i}}}{\lambda_{n_{i}}}+\nabla f_{\alpha_{n_{i}}}\left(y_{n_{i}}\right)\right\rangle \\
= & \left\langle v-l_{n_{i}}, \nabla f(v)\right\rangle \\
& -\left\langle v-l_{n_{i}}, \frac{l_{n_{i}}-x_{n_{i}}}{\lambda_{n_{i}}}+\nabla f\left(y_{n_{i}}\right)\right\rangle \\
& -\alpha_{n_{i}}\left\langle v-l_{n_{i}}, y_{n_{i}}\right\rangle \\
= & \left\langle v-l_{n_{i}}, \nabla f(v)-\nabla f\left(l_{n_{i}}\right)\right\rangle \\
& +\left\langle v-l_{n_{i}}, \nabla f\left(l_{n_{i}}\right)-\nabla f\left(y_{n_{i}}\right)\right\rangle \\
\geq & -\left\langle v-l_{n_{i}}, \nabla f\left(l_{n_{i}}\right)-\nabla f\left(y_{n_{i}}\right)\right\rangle \\
& \left.-\left\langle v-l_{n_{i}}, \frac{l_{n_{i}}-x_{n_{i}}}{\lambda_{n_{i}}}\right\rangle-\alpha_{n_{i}}, \frac{l_{n_{i}}-x_{n_{i}}}{\lambda_{n_{i}}}\right\rangle-\alpha_{n_{i}}\left\langle v-l_{n_{i}}, y_{n_{n_{i}}}\right\rangle . \\
& \langle
\end{aligned}
$$

By taking $i \rightarrow \infty$, we obtain $\langle v-\widehat{x}, w\rangle \geq 0$. Since $T$ is maximal monotone, it follows that $\hat{x} \in T^{-1} 0$ and hence $\widehat{x} \in \operatorname{VI}(C, \nabla f)$. Therefore, by Lemma $8, \widehat{x} \in \Gamma$.

Next, we show that $\widehat{x} \in F(S)$. Since $l_{n_{i}} \rightarrow \widehat{x}$ and $\| l_{n_{i}}-$ $S\left(l_{n_{i}}\right) \| \rightarrow 0$, it follows by the demiclosed principle that $\hat{x} \in$ $F(S)$. Hence, we have $\widehat{x} \in F(S) \cap \Gamma$.

Next, we show that $\widehat{x} \in \Omega$. Let $G$ be a mapping which is defined as in Lemma 6, thus we have

$$
\begin{aligned}
\left\|z_{n}-G\left(z_{n}\right)\right\| & =\left\|T_{1, \mu} T_{2, \mu} x_{n}-G\left(z_{n}\right)\right\| \\
& =\left\|G\left(x_{n}\right)-G\left(z_{n}\right)\right\| \leq\left\|x_{n}-z_{n}\right\|,
\end{aligned}
$$

and hence

$$
\begin{aligned}
\left\|x_{n}-G\left(x_{n}\right)\right\| \leq & \left\|x_{n}-z_{n}\right\|+\left\|z_{n}-G\left(z_{n}\right)\right\| \\
& +\left\|G\left(z_{n}\right)-G\left(x_{n}\right)\right\| \\
\leq & \left\|x_{n}-z_{n}\right\|+\left\|x_{n}-z_{n}\right\|+\left\|z_{n}-x_{n}\right\| \\
= & 3\left\|x_{n}-z_{n}\right\| .
\end{aligned}
$$

By taking $n \rightarrow \infty$, we have $\left\|x_{n}-G\left(x_{n}\right)\right\| \rightarrow 0$. From $\lim _{n \rightarrow \infty}\left\|x_{n}-z_{n}\right\|=0$ and $z_{n_{j}} \rightarrow \widehat{x}$, we obtain $x_{n_{j}} \rightarrow \widehat{x}$. According to demiclosedness and Lemma 6 , we have $\hat{x} \in \Omega$. Therefore, we have $\widehat{x} \in F(S) \cap \Gamma \cap \Omega$. Let $\left\{x_{n_{j}}\right\}$ be another subsequence of $\left\{x_{n}\right\}$ such that $x_{n_{j}} \rightarrow \widehat{x}$. We show that $\widehat{x}=\tilde{x}$, suppose that $\hat{x}=\tilde{x}$. Since $\lim _{n \rightarrow \infty}\left\|x_{n}-\hat{x}\right\|$ exists for all $\widehat{x} \in F(S) \cap \Gamma \cap \Omega$, it follows by the Opial's condition that

$$
\begin{aligned}
\lim _{n \rightarrow \infty}\left\|x_{n}-\hat{x}\right\| & =\liminf _{i \rightarrow \infty}\left\|x_{n_{i}}-\hat{x}\right\| \\
& <\liminf _{i \rightarrow \infty}\left\|x_{n_{i}}-\tilde{x}\right\|=\lim _{n \rightarrow \infty}\left\|x_{n}-\tilde{x}\right\| \\
& =\liminf _{j \rightarrow \infty}\left\|x_{n_{j}}-\tilde{x}\right\| \\
& <\liminf _{j \rightarrow \infty}\left\|x_{n_{j}}-\hat{x}\right\|=\lim _{n \rightarrow \infty}\left\|x_{n}-\hat{x}\right\| .
\end{aligned}
$$

It is a contradiction. Thus, we have $\widehat{x}=\tilde{x}$ and so $x_{n}-\tilde{x} \epsilon$ $F(S) \cap \Gamma \cap \Omega$. Further, from $\left\|x_{n}-y_{n}\right\| \rightarrow 0$, it follows that $y_{n} \rightarrow \tilde{x}$ and hence $z_{n}, w_{n}$. This completes the proof.

Theorem 9 extends the extragradient method according to Nadezhkina and Takahashi [35].

Corollary 10. Let $C$ be a nonempty closed convex subset in a real Hilbert space $H$. Let $F_{1}$ and $F_{2}$ be two bifunctions from $C \times$ $C \rightarrow \mathbb{R}$ satisfying (A1)-(A4). Let $\mu>0$ and let $T_{1, \mu}$ and $T_{2, \mu}$ be defined as in Lemma 5 associated to $F_{1}$ and $F_{2}$, respectively. Let $S$ be a quasi-nonexpansive mapping of $C$ into itself such that $F(S) \cap \Gamma \cap \Omega \neq \emptyset$. Let $\left\{x_{n}\right\},\left\{y_{n}\right\},\left\{z_{n}\right\}$, and $\left\{w_{n}\right\}$ be the sequence in $C$ generated by the following extragradient algorithm:

$$
\begin{gathered}
x_{0}=x \in C, \\
w_{n} \in C ; \quad F_{2}\left(w_{n}, z\right)+\frac{1}{\mu}\left\langle z-w_{n}, w_{n}-x_{n}\right\rangle \geq 0, \quad \forall z \in C, \\
z_{n} \in C ; \quad F_{1}\left(z_{n}, z\right)+\frac{1}{\mu}\left\langle z-z_{n}, z_{n}-w_{n}\right\rangle \geq 0, \quad \forall z \in C, \\
y_{n}=P_{C}\left(I-\lambda_{n} \nabla f\right) z_{n}, \\
x_{n+1}=\beta_{n} x_{n}+\left(1-\beta_{n}\right) S P_{C}\left(x_{n}-\lambda_{n} \nabla f\left(y_{n}\right)\right),
\end{gathered}
$$

where $\sum_{n=0}^{\infty} \alpha_{n}<\infty,\left\{\lambda_{n}\right\} \subset[a, b]$ for some $a, b \in\left(0,1 /\|A\|^{2}\right)$ and $\left\{\beta_{n}\right\} \subset[c, d]$ for some $c, d \in(0,1)$. Then, the sequences $\left\{x_{n}\right\}$ and $\left\{y_{n}\right\}$ converge weakly to an element $\widehat{x} \in F(S) \cap \Gamma \cap \Omega$.

Corollary 11. Let $C$ be a nonempty closed convex subset in a real Hilbert space $H$. Let $F_{1}$ and $F_{2}$ be two bifunctions from $C \times$ 
$C \rightarrow \mathbb{R}$ satisfying (A1)-(A4). Let $\mu>0$ and let $T_{1, \mu}$ and $T_{2, \mu}$ be defined as in Lemma 5 associated to $F_{1}$ and $F_{2}$, respectively. Let $S$ be a quasi-nonexpansive mapping of $C$ into itself such that $F(S) \cap \Gamma \cap \Omega \neq \emptyset$. Let $\left\{x_{n}\right\},\left\{y_{n}\right\}$, and $\left\{z_{n}\right\}$ be the sequence in $C$ generated by the following extragradient algorithm:

$$
\begin{gathered}
x_{0}=x \in C, \\
z_{n} \in C ; \quad F_{1}\left(z_{n}, z\right)+\varphi(z)-\varphi\left(z_{n}\right) \\
+\frac{1}{\mu}\left\langle z-z_{n}, z_{n}-x_{n}\right\rangle \geq 0, \quad \forall z \in C, \\
y_{n}=P_{C}\left(I-\lambda_{n} \nabla f_{\alpha_{n}}\right) z_{n}, \\
x_{n+1}=\beta_{n} x_{n}+\left(1-\beta_{n}\right) S P_{C}\left(x_{n}-\lambda_{n} \nabla f_{\alpha_{n}}\left(y_{n}\right)\right),
\end{gathered}
$$

where $\sum_{n=0}^{\infty} \alpha_{n}<\infty,\left\{\lambda_{n}\right\} \subset[a, b]$ for some $a, b \in\left(0,1 /\|A\|^{2}\right)$ and $\left\{\beta_{n}\right\} \subset[c, d]$ for some $c, d \in(0,1)$. Then, the sequences $\left\{x_{n}\right\}$ and $\left\{y_{n}\right\}$ converge weakly to an element $\widehat{x} \in F(S) \cap \Gamma \cap \Omega$.

Proof. Setting $F_{2}=0$ in Theorem 9, we obtain the desired result.

Corollary 12. Let $C$ be a nonempty closed convex subset in a real Hilbert space $H$. Let $S$ be a quasi-nonexpansive mapping of $C$ into itself such that $F(S) \cap \Gamma \neq \emptyset$. Let $\left\{x_{n}\right\},\left\{y_{n}\right\}$, and $\left\{z_{n}\right\}$ be the sequence in $C$ generated by the following extragradient algorithm:

$$
\begin{gathered}
x_{0}=x \in C, \\
y_{n}=P_{C}\left(I-\lambda_{n} \nabla f_{\alpha_{n}}\right) x_{n}, \\
x_{n+1}=\beta_{n} x_{n}+\left(1-\beta_{n}\right) S P_{C}\left(x_{n}-\lambda_{n} \nabla f_{\alpha_{n}}\left(y_{n}\right)\right),
\end{gathered}
$$

where $\sum_{n=0}^{\infty} \alpha_{n}<\infty,\left\{\lambda_{n}\right\} \subset[a, b]$ for some $a, b \in\left(0,1 /\|A\|^{2}\right)$ and $\left\{\beta_{n}\right\} \subset[c, d]$ for some $c, d \in(0,1)$. Then, the sequences $\left\{x_{n}\right\}$ and $\left\{y_{n}\right\}$ converge weakly to an element $\widehat{x} \in F(S) \cap \Gamma$.

Proof. Setting $F_{1}=F_{2}=0$ in Theorem 9, we obtain the desired result.

\section{Acknowledgments}

The authors would like to thank The Commission on Higher Education for financial support. Moreover, K. Sombut is also supported by The Strategic Scholarships Fellowships Frontier Research Networks under Grant CHE-Ph.D-THASUP/86/2550, Thailand.

\section{References}

[1] W. R. Mann, "Mean value methods in iteration," Proceedings of the American Mathematical Society, vol. 4, pp. 506-510, 1953.

[2] W. G. Dotson Jr., "On the Mann iterative process," Transactions of the American Mathematical Society, vol. 149, pp. 65-73, 1970.

[3] S. Plubtieng, R. Wangkeeree, and R. Punpaeng, "On the convergence of modified Noor iterations with errors for asymptotically nonexpansive mappings," Journal of Mathematical Analysis and Applications, vol. 322, no. 2, pp. 1018-1029, 2006.
[4] S. Plubtieng and R. Wangkeeree, "Strong convergence theorems for multi-step Noor iterations with errors in Banach spaces," Journal of Mathematical Analysis and Applications, vol. 321, no. 1, pp. 10-23, 2006.

[5] E. Blum and W. Oettli, "From optimization and variational inequalities to equilibrium problems," The Mathematics Student, vol. 63, no. 1-4, pp. 123-145, 1994.

[6] D. S. Flam and A. S. Antipin, "Equilibrium programming using proximal-like algorithms," Mathematical Programming, vol. 78, no. 1, pp. 29-41, 1997.

[7] A. Moudafi, "Second-order differential proximal methods for equilibrium problems," Journal of Inequalities in Pure and Applied Mathematics, vol. 4, no. 1, article 18, 2003.

[8] S. Takahashi and W. Takahashi, "Viscosity approximation methods for equilibrium problems and fixed point problems in Hilbert spaces," Journal of Mathematical Analysis and Applications, vol. 331, no. 1, pp. 506-515, 2007.

[9] W. Takahashi and K. Zembayashi, "Strong convergence theorem by a new hybrid method for equilibrium problems and relatively nonexpansive mappings," Fixed Point Theory and Applications, vol. 2008, Article ID 528476, 11 pages, 2008.

[10] S. Plubtieng and W. Sriprad, "A viscosity approximation method for finding common solutions of variational inclusions, equilibrium problems, and fixed point problems in Hilbert spaces," Fixed Point Theory and Applications, vol. 2009, Article ID 567147, 20 pages, 2009.

[11] S. Plubtieng and T. Thammathiwat, "A viscosity approximation method for equilibrium problems, fixed point problems of nonexpansive mappings and a general system of variational inequalities," Journal of Global Optimization, vol. 46, no. 3, pp. 447-464, 2010.

[12] A. Moudafi, "From alternating minimization algorithms and systems of variational inequalities to equilibrium problems," Communications on Applied Nonlinear Analysis, vol. 16, no. 3, pp. 31-35, 2012.

[13] Y. Yao, Y.-C. Liou, and J.-C. Yao, "New relaxed hybridextragradient method for fixed point problems, a general system of variational inequality problems and generalized mixed equilibrium problems," Optimization, vol. 60, no. 3, pp. 395-412, 2011.

[14] S. Plubtieng and K. Sombut, "Weak convergence theorems for a system of mixed equilibrium problems and nonspreading mappings in a Hilbert space," Journal of Inequalities and Applications, vol. 2010, Article ID 246237, 12 pages, 2010.

[15] Y. Censor and T. Elfving, "A multiprojection algorithm using Bregman projections in a product space," Numerical Algorithms, vol. 8, no. 2-4, pp. 221-239, 1994.

[16] C. Byrne, "Iterative oblique projection onto convex sets and the split feasibility problem," Inverse Problems, vol. 18, no. 2, pp. 441453, 2002.

[17] H.-K. Xu, "Iterative methods for the split feasibility problem in infinite-dimensional Hilbert spaces," Inverse Problems, vol. 26, no. 10, article 105018, 2010.

[18] Y. Censor, T. Elfving, N. Kopf, and T. Bortfeld, “The multiplesets split feasibility problem and its applications for inverse problems," Inverse Problems, vol. 21, no. 6, pp. 2071-2084, 2005.

[19] Y. Censor, A. Motova, and A. Segal, "Perturbed projections and subgradient projections for the multiple-sets split feasibility problem," Journal of Mathematical Analysis and Applications, vol. 327, no. 2, pp. 1244-1256, 2007. 
[20] C. Byrne, "A unified treatment of some iterative algorithms in signal processing and image reconstruction," Inverse Problems, vol. 20, no. 1, pp. 103-120, 2004.

[21] B. Qu and N. Xiu, "A note on the CQ algorithm for the split feasibility problem," Inverse Problems, vol. 21, no. 5, pp. 1655$1665,2005$.

[22] H.-K. Xu, "A variable KrasnoselskiiMann algorithm and the multiple-set split feasibility problem," Inverse Problems, vol. 22, no. 6, pp. 2021-2034, 2006.

[23] Q. Yang, "The relaxed CQ algorithm solving the split feasibility problem," Inverse Problems, vol. 20, no. 4, pp. 1261-1266, 2004.

[24] J. Zhao and Q. Yang, "Several solution methods for the split feasibility problem," Inverse Problems, vol. 21, no. 5, pp. 17911799, 2005.

[25] Y. Yao, R. Chen, G. Marino, and Y. C. Liou, "Applications of fixed-point and optimization methods to the multiple-set split feasibility problem," Journal of Applied Mathematics, vol. 2012, Article ID 927530, 21 pages, 2012.

[26] X. Yu, N. Shahzad, and Y. Yao, "Implicit and explicit algorithms for solving the split feasibility problem," Optimization Letters, vol. 6, no. 7, pp. 1447-1462, 2012.

[27] B. Eicke, "Iteration methods for convexly constrained ill-posed problems in Hilbert space," Numerical Functional Analysis and Optimization, vol. 13, no. 5-6, pp. 413-429, 1992.

[28] L. Landweber, "An iteration formula for Fredholm integral equations of the first kind," American Journal of Mathematics, vol. 73, pp. 615-624, 1951.

[29] L.-C. Ceng, Q. H. Ansari, and J.-C. Yao, "An extragradient method for solving split feasibility and fixed point problems," Computers \& Mathematics with Applications, vol. 64, no. 4, pp. 633-642, 2012.

[30] D. P. Bertsekas and E. M. Gafni, "Projection methods for variational inequalities with application to the traffic assignment problem," Mathematical Programming Study, no. 17, pp. 139-159, 1982.

[31] D. Han and H. K. Lo, "Solving non-additive traffic assignment problems: a descent method for co-coercive variational inequalities," European Journal of Operational Research, vol. 159, no. 3, pp. 529-544, 2004.

[32] J.-W. Peng and J.-C. Yao, "A new hybrid-extragradient method for generalized mixed equilibrium problems, fixed point problems and variational inequality problems," Taiwanese Journal of Mathematics, vol. 12, no. 6, pp. 1401-1432, 2008.

[33] M. O. Osilike, S. C. Aniagbosor, and B. G. Akuchu, "Fixed points of asymptotically demicontractive mappings in arbitrary Banach spaces," Panamerican Mathematical Journal, vol. 12, no. 2, pp. 77-88, 2002.

[34] R. T. Rockafellar, "On the maximality of sums of nonlinear monotone operators," Transactions of the American Mathematical Society, vol. 149, pp. 75-88, 1970.

[35] N. Nadezhkina and W. Takahashi, "Weak convergence theorem by an extragradient method for nonexpansive mappings and monotone mappings," Journal of Optimization Theory and Applications, vol. 128, no. 1, pp. 191-201, 2006. 


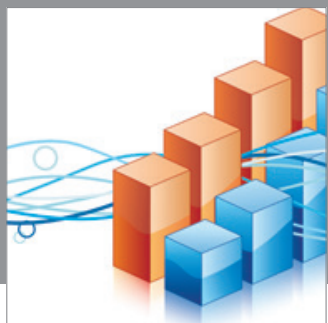

Advances in

Operations Research

mansans

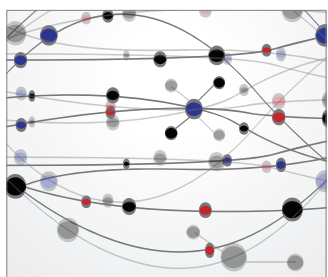

The Scientific World Journal
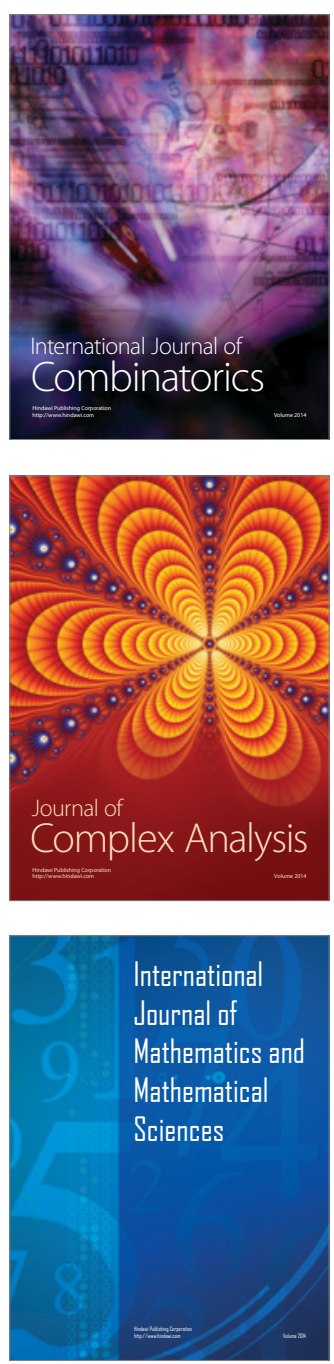
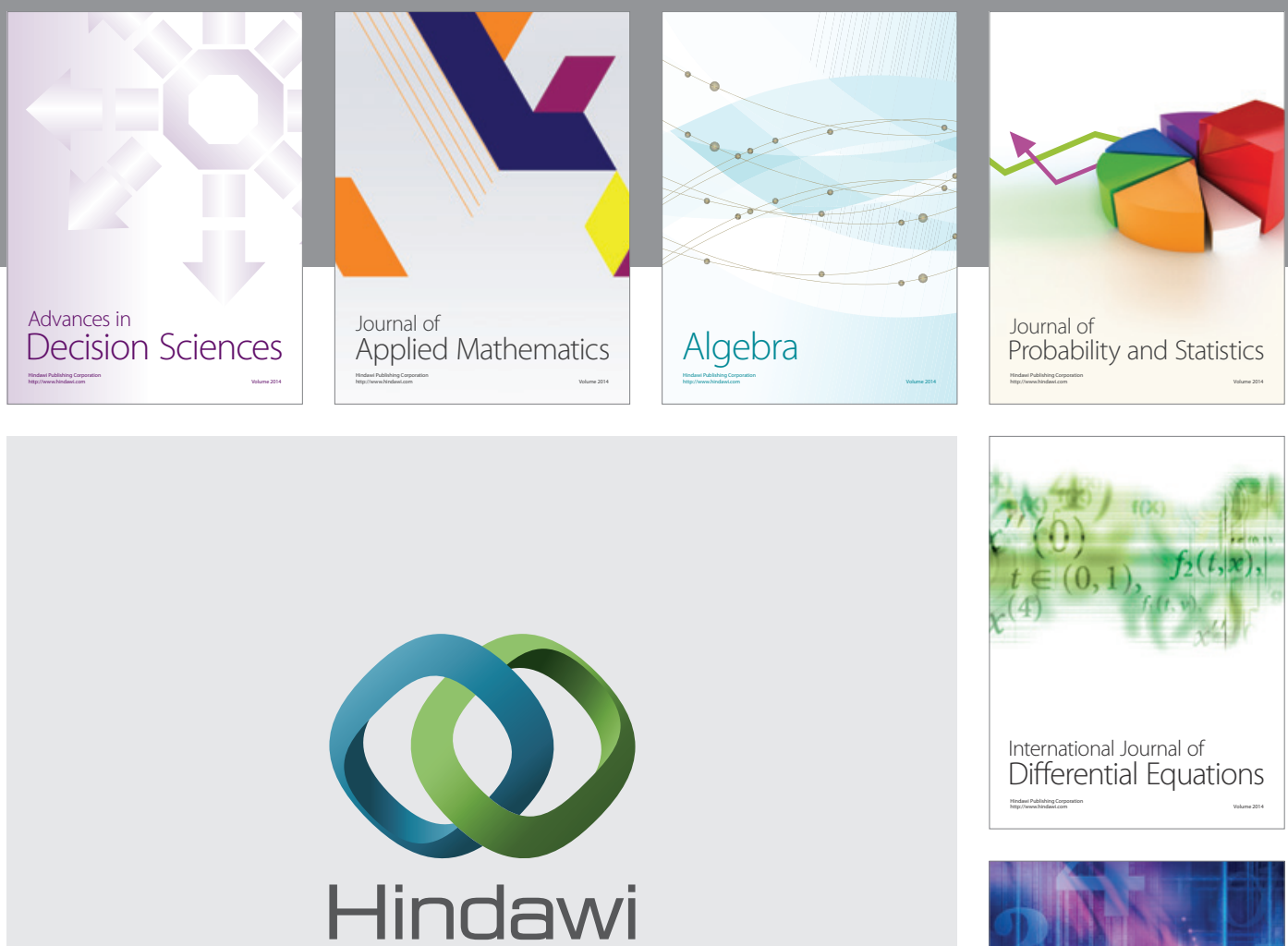

Submit your manuscripts at http://www.hindawi.com
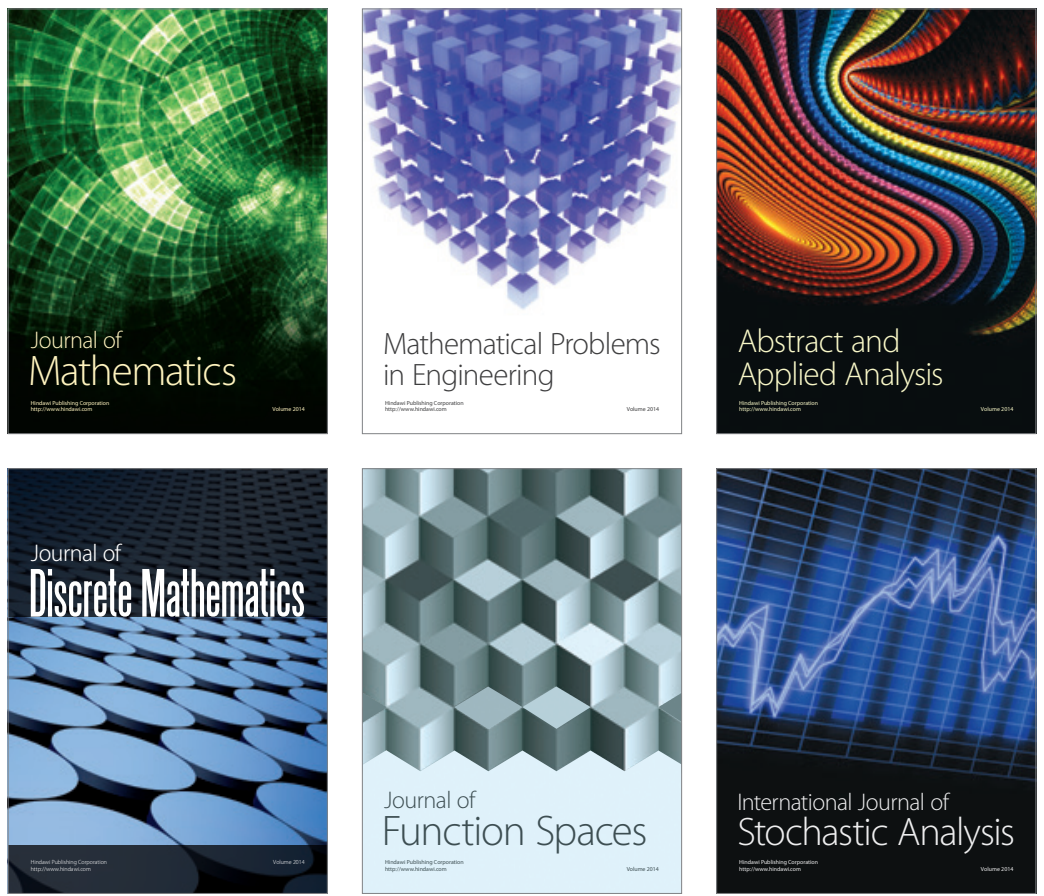

Journal of

Function Spaces

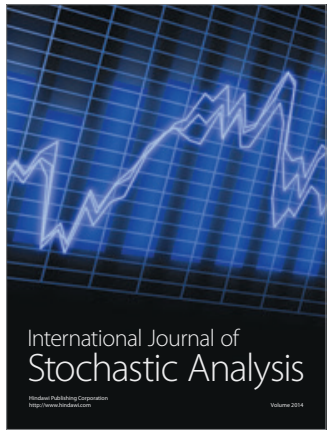

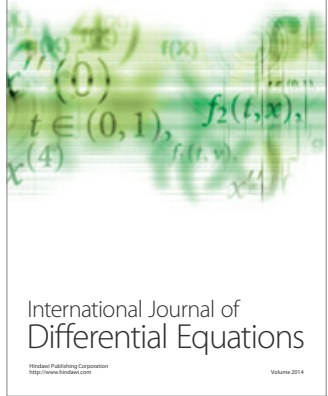
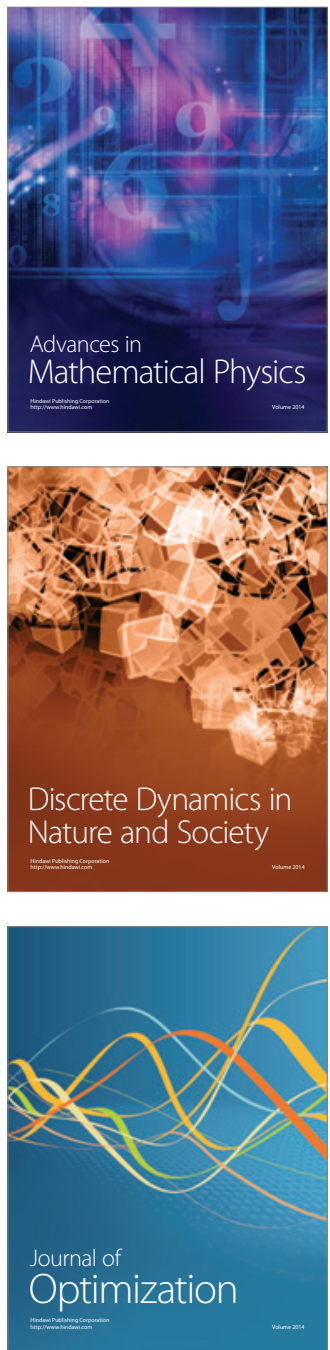